\title{
Detailed geometrical information of aircraft fuel tanks incorporated into fuel system simulation models
}

\author{
Ingela Lind \\ Alexandra Oprea \\ SAAB Aeronautics \\ SE 58188 Linköping, Sweden \\ ingela.lind@saabgroup.com alexandra.oprea@saabgroup.com
}

\begin{abstract}
Fuel tanks in fighter aircraft have an irregular shape which is given by a detailed CAD model. To simulate a fuel system with sufficient amount of detail to solve the design issues, necessary geometrical information need to be given in a compact and computationally fast form. A function approximation using radial basis functions is suggested and compared with some other methods. The complete process from production scale CAD model to system simulation model is considered.
\end{abstract}

Keywords: aircraft design; fuel systems simulation; geometrical representation; surrogate model; radial basis functions

\section{Introduction}

A fighter aircraft fuel system is a system of many parts. Fuel fills up large parts of the aircraft not occupied with other equipment and the many different systems of the aircraft often pass through the tanks. To keep control of the center of gravity the tanks are divided into smaller parts and are interconnected by pipes. Fuel is pumped between the tanks to a collector tank which has a negative g-load compartment to enable the aircraft to fly inverted. The tanks are also pressurized to avoid evaporation of the fuel at high altitudes.

When designing aircraft fuel systems several issues demand detailed simulation models for analysis. The most important are

- Is it possible to provide the engine with fuel with enough pressure regardless of what pilot maneuvers and equipment faults that occur?

- Can the amount of accessible fuel be correctly determined at all times?
- Can the structural strength of the hull and all installation parts be estimated with good precision?

When these problems are solved, questions related to optimization of weight, fuel consumption, and heat storing capabilities as well as other issues need to be considered.

The fuel system simulation models needed to describe the system tend to be large ( 400 state variables, 16000 time-varying variables) due to the high number of parts involved. The combination of fuel (incompressible fluid) and pressurization air (compressible fluid) and the necessity to handle both fast time constants (as when a tank get full) and slow time constants (heat storage) make the models stiff and a bit awkward to work with. Still, the information gained from using the simulation models more than pays off the work spent to keep the models executable and is seen as a prerequisite for successful fuel system design work at Saab Aeronautics. A theoretical background on fuel system design can be found in [1] and how Dymola and other simulation tools are used in the system design process is described in [2] and [3].

In the past few years the idea of bringing geometrical information from CAD models into simulation models has gone from a distant dream to something actually achievable. To investigate if it was possible to get better accuracy of the fuel tank representation in the simulation models used, a study [4] was made to show the feasibility of extracting geometrical information from CAD models, do a function approximation of the data and then use the function in a tank model in Dymola. The work is not yet finalized to the level of inclusion in production processes, but the major steps and an evaluation of results are done. The intention is that the accuracy of the simulation models shall meet the measurement precision demands on the aircraft, and to improve the efficiency of the loads computations while simulation times are kept at the same level as before. 
This can be done using two types of model improvements. First, the geometrical representation of the fuel tanks is changed from a simple twodimensional square box to a full three dimensional representation of the complex geometry. Next, the aircraft model is changed from a point model where all accelerations act on the same point to a rigid body where accelerations in each tank depend on both the accelerations in the aircraft's center of gravity and the torque acting around it.

In this paper the major steps of the procedure will be discussed as well as later results showing that the process [4] can be scaled to full production size CATIA models as well as full size fuel simulation models.

\section{Assumptions}

A typical fighter plane fuel tank has a complicated shape; an example is shown in Figure 1. It is made up of many non-convex surfaces and even internal parts where bulky equipment is immersed in fuel. Due to the high order of complexity, describing the details of the fuel tank geometry in a simulation model is not feasible at the moment.



Figure 1 Typical body fuel tank shape, which is one of several different basic shapes. Note that equipment is immersed in fuel and fills up space within the tank.

It is assumed that the case is semi-static, such that the fuel surface is perpendicular to the acceleration vector of the tank at each time instant and that there is no fuel sloshing. Although a bit contradictory, it is also assumed that the fuel undergoes enough sloshing so that all fuel surfaces of the different compartments within the same tank are at exactly the same height, as if they were connected beneath the surface (which often is the case).
The information needed to perform a fuel system simulation is, given the orientation of the acceleration vector, the center of gravity of the fuel in a particular tank and the position of a point on the fuel surface. Both these vectors depend on the volumetric amount of fuel in the tank which is connected to the fuel mass state variable through the temperature dependent density. This means that it is enough to have a function that transforms the four variables fuelVolume and the 3D acceleration vector to the six variables given by the two 3D vectors representing the point on the fuel's surface and the fuel's center of gravity. The means to represent this function could be a table, but the assumption has been made that a function approximation would be both faster and less memory consuming.

\section{Geometric data transformation}

The first prerequisite for a fuel system simulation is the extraction of the geometric data from CATIA.

\subsection{Extraction of geometric information}

Each fuel tank in the Jas 39 Gripen has its own CAD model, and an analysis extracting the center of gravity $(\mathrm{CG})$ and fuel surface data needs to be performed on each of them. The analysis itself consists of a macro written in VBScript. An early version of it can be found in [4]. In a nutshell, the steps of the analysis are as follows:

- Transforming the original fuel tank into a "dumb" solid, without construction history or identifiable individual features. This is done in order to reduce the file size, which affects the analysis time considerably.

- Creating the 2 bodies for the analysis: a "reference" body for comparison purposes and a "fuel" body on which the actual analysis will be performed. The dimensions of the "reference" body are recorded and saved.

- Creating the required elements for the analysis: the acceleration vector line, the sectioning plane perpendicular to the line which will split the "fuel" body in steps of pre-set length and the measurements on the "fuel" body which will update every time the body is sectioned.

- Performing the actual analysis. For each acceleration vector orientation in the aircraft's maneuverable range, the fuel body is split in steps of around 10 liters. For each split the 
values of the resulting fuel volume, center of gravity and of the position of a point on the fuel's surface are saved to a text file.

\subsection{Function approximation of data}

The CATIA step generates around 20,000 to 40,000 distinct data entries for each fuel tank, with 10 parameters each. Although numerous, these are not enough since the simulation needs the CG and surface point coordinates for other acceleration vectors and volumes, as well. Therefore, a data interpolation step is required. To do the interpolation using Dymola interpolation tables would be feasible with a small amount of data in a low-dimensional case, but the high dimension and the amount of data calls for other methods. In this case the data interpolation is done using a parameterized function approximation called radial basis functions (RBF) networks. They are thought to be one of the best ways of approximation multi-variate scattered data, due to their excellent approximation properties [5], although in some cases vulnerable to the Runge phenomenon [8]. In short, they can be visualized as an "input - process output" system. The input is the data generated from CATIA - the $X, Y, Z$ orientations of the acceleration vector, the fuel volume, the $X, Y, Z$ coordinates of the fuel surface and the corresponding ones of the tank's center of gravity. The output is a function, $s$, which can give a good approximation of the data for inputs different than the ones where the value of the exact function is known. The approximating function is defined using fewer points than the ones available in the input data (points which will be called centers). This means that a data reduction with maintained generalization ability is done. For visualization, see Figure

\section{2 .}

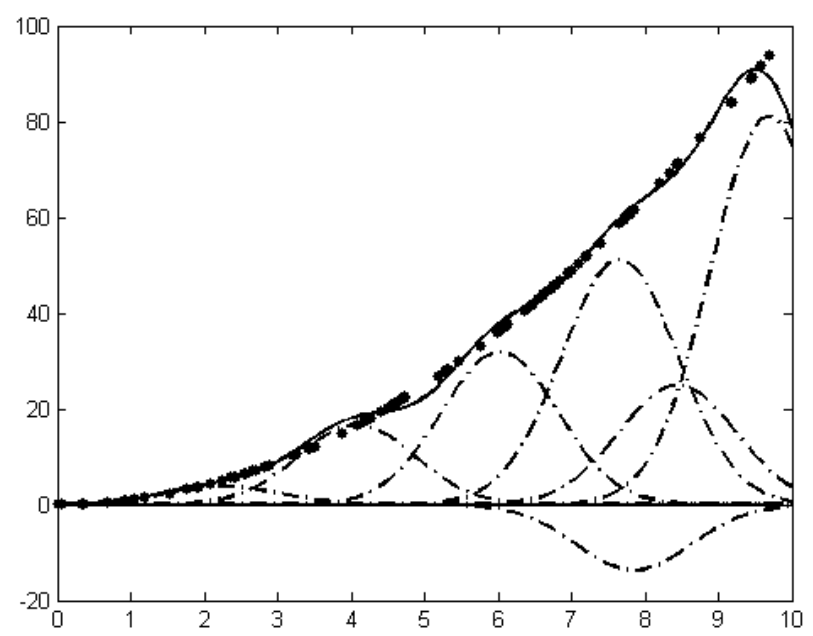

Figure 2 The Gaussian functions at the selected centers (dash-dotted) and the resulting approximating function (solid) based on the input data (points). In this plot, the Runge phenomenon is visible at the right edge of the interval (the solid line drops), but it might also be a part of the explanation for the drop of the curve around $x=5$.

The middle layer, the so-called "process", is defined using the RBF functions themselves. The approximating function $s(x)=W_{1}+\sum_{i=2 \ldots n} W_{i} f\left(k\left\|x_{i}-x\right\|\right)$ is given by a weighted sum of a uni-variate function $f$ with the Euclidian distance between the $x_{i}$ and $x$ as argument, where $x$ are the points at which the approximation function is calculated, and $x_{i}$ are the centers with respect to which the function is defined. The weighting factor $W_{i}$ is associated to each center $x_{i}$. It is determined such that the error between the approximating function and the input data is minimized. The scaling factor $k$ influences the support area of the function $f$.

The centers are selected using the orthogonal forward regression algorithm, presented in [6] and [7]. They could be selected at random, but the algorithm uses the modified Gram-Schmidt orthogonalization procedure to select the centers which minimize the error in the least-squares sense. The benefit of using the modified Gram-Schmidt method is that the resulting approximation is sparse in parameters. It starts from a large set of potential centers - basically, all the data resulting from the CATIA analysis, filtering them until the error sinks below a specified threshold. To be able to handle the very large data sets an approach where the data sets are divided into smaller pieces and the results recomputed several times is used, see [4]. When all data is processed, the algorithm returns the selected centers and their corresponding weights and writes them to a file to be used in the dynamic simulation in Dymola.

All RBF networks work according to the same principles. The differing factor is the function $f$, which makes up the linear combination defining the approximating function $s$. The ideal for the fuel tank problem, which is local and almost smooth in its nature, is to have a function with local support, so that new input data would not influence points situated far from it. Several possible functions have been tested to determine which ones are good choices with respect to sparseness of the parameters in the approximation and computational speed in the Dymola implementation. The typical number of parameters of the approximation is in the range 300 to 3000 , a data reduction of a factor 100 to 1000 . 
A standard choice of a Gaussian function, see Figure 2 , seems to fit the fuel tank problem best. There were concerns regarding computational effectiveness in Dymola as the exponential function is not considered cheap so other choices were considered.

Using $r=\left\|x_{i}-x\right\|$, the investigated options were

- the inverse quadratic function,

$$
f(r)=\frac{1}{1+(k r)^{2}}
$$

- the inverse multiquadric function,

$$
f(r)=\frac{1}{\sqrt{1+(k r)^{2}}}
$$

- the rational quadratic, $f(r)=1-\frac{r^{2}}{1+r^{2}}$

- Wendland's functions,

$$
f(r)=\left\{\begin{array}{c}
(1-r)^{5}(5 r+1), \quad r<1(\text { or } 0.5) \\
0, \quad \text { otherwise }
\end{array}\right.
$$

They all give less sparse results and need both more memory to store parameters and more multiplications to compute $s(x)$. Also B-splines outside a RBF framework have been considered, but do not fit ideally to high-dimensional non-latticed data.

\subsection{Tank model implementation}

Implementing the radial basis functions in the existing tank model of the commercial library AircraftFluidSystems (developed mainly by Modelon $A B$ and partly by Saab Aeronautics) was a simple task. The only modification needed was the replacement of the existing distance computation between the position of the fuel surface and the tank ports of the connected pipes. This is now done using a scalar product and the approximating function $s(x)$.

The change in the acceleration vector definition brought by viewing the aircraft as a rigid body instead of a point mass was also straightforward.

\section{Results}

\subsection{Influence on accuracy}

A comparison between two fuel system simulations performed using the old two-dimensional tank representation and two simulations incorporating the new three-dimensional representation is presented in [4]. Both the 2D and 3D simulations are performed for two levels of accuracy of the CATIA analysis. The results show that there is only a minor difference in the system simulation precision between the two
CATIA target sampling accuracies of 18 and 12 liters. Compared to the CAD measurements of three different points for several acceleration vectors, the simulations resulted in an error of $3.3 \mathrm{~kg}$ for the 181 precision (with a maximum error of $10 \mathrm{~kg}$ ) and a 3.1 $\mathrm{kg}$ error for the 121 precision (with a maximum error of $6 \mathrm{~kg}$ ). For the two-dimensional tank representation the same average difference is $42 \mathrm{~kg}$ (with a maximum of $89 \mathrm{~kg}$ ). It then follows that a threedimensional representation of a moderate size makes a large improvement in the simulation accuracy of the fuel measurements. Comparison with a real fuel tank is not achievable since the tanks are not yet built, but it has been shown earlier that 'fuel measurements' in CAD models correspond well with fuel sensor calibration measurements in built tanks.

This accuracy improvement affects all parts of the fuel system simulation model, as the fuel flow through pumps and pipes depends on the fuel level in each tank.

\subsection{Influence on simulation times}

A comparison between the different possible kernel functions revealed that the initial choice of the Gaussian was the correct one. The evaluated functions, along with their training, computation times in MATLAB and simulation times in Dymola for a simple test model are given in Table 1. All the simulations are performed on the same tank, with the same parameters (the scaling factor $k=0.8$ where necessary and the error threshold set at $5 \mathrm{~mm}$ ). The MATLAB computation time take only computation of the function $s(x)$ into account, while the Dymola simulation time also involves computations of all other equations necessary for a tank, two pipes and two sources in a test case.

Table 1 Comparison of training and simulation times for different kernel functions

\begin{tabular}{|c|c|c|c|}
\hline Function & $\begin{array}{c}\text { Training } \\
\text { time, } \mathrm{s}\end{array}$ & $\begin{array}{c}\text { MATLAB } \\
\text { computation } \\
\text { time, } \mathrm{s}\end{array}$ & $\begin{array}{c}\text { Dymola } \\
\text { simulation } \\
\text { time, } \mathrm{s}\end{array}$ \\
\hline Gaussian & 36.63 & 0.84 & 157 \\
\hline $\begin{array}{c}\text { Inverse } \\
\text { quadratic }\end{array}$ & 58.85 & 1.12 & 190 \\
\hline $\begin{array}{c}\text { Inverse mul- } \\
\text { tiquadric }\end{array}$ & 139.26 & 4.15 & 161 \\
\hline $\begin{array}{c}\text { Rational } \\
\text { quadratic }\end{array}$ & 63.57 & 1.11 & 155 \\
\hline $\begin{array}{c}\text { Wendland, } \mathrm{r} \\
<1.0 \mathrm{~mm}\end{array}$ & 218.13 & 2.93 & 158 \\
\hline $\begin{array}{c}\text { Wendland, } \mathrm{r} \\
<0.5 \mathrm{~mm}\end{array}$ & 318.08 & 2.95 & 176 \\
\hline
\end{tabular}



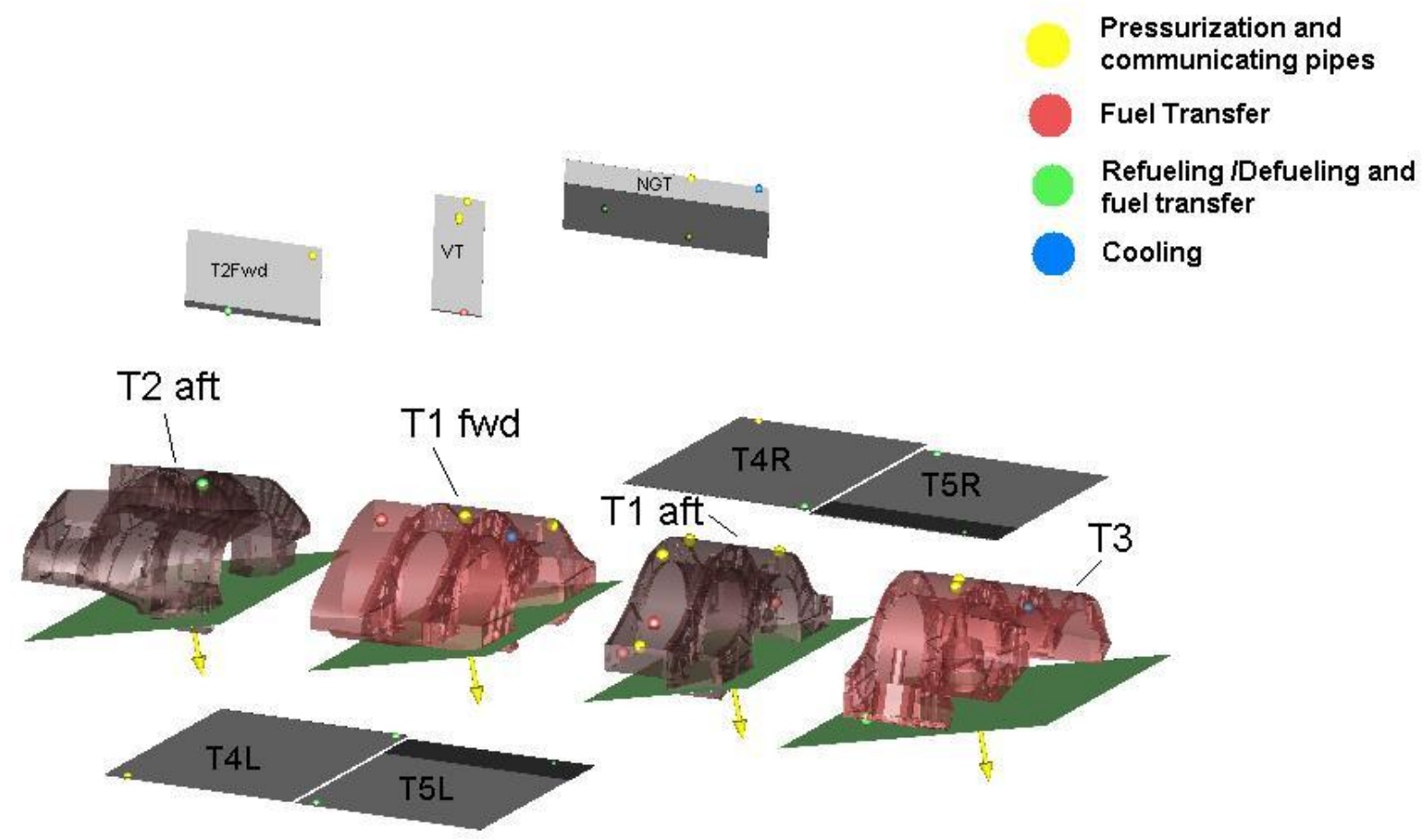

Figure 3 Visualization/animation of the fuel system using graphics from CATIA and simulation results from the fuel system simulation model. The green and black surfaces show the fuel level in the tanks. The yellow arrows show the acceleration vector for each tank and the colored balls show inlets and outlets of pipes for different purposes.

As for the variation of the Gaussian function with its defining parameters, simulations showed that there are optimum values of the scaling factor and of the error threshold. Any values different from these optimal ones lead to extended simulation times, without a significant improvement in accuracy. For the simulations with an error value of $0.01 \mathrm{~mm}$, the MATLAB training time was between 3000 and $4000 \mathrm{~s}$, while for errors of $1 \mathrm{~mm}$ it dropped to several hundreds of seconds. The Dymola simulation results are summarized in Table 2. If the Runge phenomenon influences the function approximation, the simulation times easily grow a factor 10 or more, so care must be taken to avoid it.

Table 2 Dymola simulation times for different Gaussian settings

\begin{tabular}{|l|l|}
\hline Settings & Dymola simulation time, $\mathrm{s}$ \\
\hline $\mathrm{k}=0.05$, err $=0.01$ & 166 \\
\hline $\mathrm{k}=0.4$, err $=0.01$ & 182 \\
\hline $\mathrm{k}=0.8$, err $=0.01$ & 177 \\
\hline
\end{tabular}

When a complete fuel system simulation model with four three-dimensional tank representations is compared to the same model having two-dimensional tank representations the times in Table 3 are achieved. The translation/compilation time depends strongly on the number of defining parameters required by the function approximation, which is a reason to use sparse representations. The simulation time only grows by a factor 3 , which is considered to be successful, given the higher accuracy of the results. The comparison case is representative of a typical simulation analysis.

Table 3 Comparison of simulation times

\begin{tabular}{|l|l|l|}
\hline & $\begin{array}{l}\text { Translation/compilation } \\
\text { time }(\mathrm{min})\end{array}$ & $\begin{array}{l}\text { Simulation } \\
\text { time }(\mathrm{min})\end{array}$ \\
\hline 2D tank & 1.5 & 10 \\
\hline 3D tank & 10.5 & 30 \\
\hline
\end{tabular}

\subsection{Influence on system insight}

An animation of the tank models was implemented in order to identify what can be achieved, see Figure 4. This addition proved to be worthwhile from the very first simulations. The insight into the system behavior it provides shortens the time to learn the system. It is also a fast means of finding errors. For instance, one of the easiest errors to perform and most difficult to find is orienting the acceleration vector the wrong way. This can easily occur since 
different departments use different coordinate systems and boundary conditions for simulations have many different sources. But in the animation this error is easily detected.

\section{Conclusions}

A full geometrical representation of fuel tanks at a given accuracy tailor made to accommodate fuel system simulation is no more a distant dream but a fully achievable task. The work has shown that

- it is possible to achieve an appropriate level of accuracy for all intended design studies,

- it is important to get a sparse representation (to keep the translation/compilation time down),

- several different choices of radial basis functions are usable and that the Gaussian is comparable to the others with respect to simulation time, but give more sparse representations,

- care is needed to avoid the Runge phenomenon [8] (which may slow down simulations considerably when the fuel level is close to a pipe end), and

- using RBF as function approximation keeps simulation times in the same level of magnitude as the simple and much less accurate 2D square box approximation previously used.

\section{Acknowledgements}

Jonas Wikström (Linköpings University) has dedicated his master's project to this project at Saab Aeronautics.

Sara Ekermann (Linköpings University) has worked with visualization and large-scale testing of results.

Thanks to Dassault Systémes who has provided an evaluation license for the work in Catia as well as acted discussion partner of what is achievable.

\section{References}

[1] Gavel. H. (2007) On Aircraft Fuel Systems - Conceptual Design and Modeling. Dissertation No.1067, Division of Machine Design, Department of Mechanical Engineering, Linköpings University. ISBN 97891-85643-04-2
[2] Lind. I. \& Andersson. H. (2011) Model Based Systems Engineering for Aircraft Systems - How does Modelica Based Tools Fit? In proceedings of the 8th International Modelica Conference, Dresden, 2011

[3] Steinkellner S., Andersson H., Gavel H. and Krus P. Modeling and simulation of Saab Gripen's vehicle systems, AIAA Modeling and Simulation Technologies Conference, Chicago, USA, AIAA 20096134, 2009

[4] Wikström J., 3D Model of Fuel Tank for System Simulation: A methodology for combining CAD models with simulation tools, Masters thesis LIU-IEI-TEK-A11/01201-SE, Linköpings University, 2011,

http://urn.kb.se/resolve?urn=urn:nbn:se:li u:diva-71370

[5] Buhmann, M. D. Radial Basis Functions, Acta Numerica (2000) 1-38.

[6] Chen. S., Billings. S.A. \& Lou. W. (1989) Orthogonal least squares methods and their application to non-linear system identification. Internal Journal of Control, 50:5, $1873-1896$

[7] Chen. S., Billings. S.A., Cowan. C.F.N. \& Grant. P.M. (1990) Practical identification of NARMAX models using radial basis functions. Internal Journal of Control, 52:6, $1327-1350$

[8] Boyd, J.P., Six strategies for defeating the Runge Phenomenon in Gaussian radial basis functions on a finite interval. Computers and Mathematics with Applications, 60 (2010), 3108-3122. 